\title{
Complexes of fluorinated, silicone and hydrocarbon surfactants with carboxymethylcellulose and their influence on properties of the alumina suspension
}

\author{
Jakub Matusiak $^{1}$ - Elżbieta Grządka ${ }^{1} \cdot$ Małgorzata Paszkiewicz $^{1}$ - Jacek Patkowski ${ }^{1}$
}

Received: 4 January 2019 / Revised: 26 February 2019 / Accepted: 28 February 2019 / Published online: 30 March 2019

(C) The Author(s) 2019

\begin{abstract}
The aim of this study was to investigate the influence of different types of surfactants on the adsorption, stability, and electrokinetic properties of the carboxymethylcellulose/alumina system as well as the interactions between the surfactants and the polymer. The authors decided to use fluorinated and silicone surfactants besides the conventional hydrocarbon ones. The obtained results showed that carboxymethylcellulose $(\mathrm{cmc})$ adsorption increased in the presence of surfactants due to the formation of polymer-surfactant complexes (the increase of the adsorption from about $0.020 \mathrm{mg} \mathrm{m}^{-2}$ for pure cmc to $0.030 \mathrm{mg} \mathrm{m}^{-2}$ for MTAB/cmc and SJ2/cmc mixtures). The obtained complexes of $0.00336 \% \mathrm{MTAB} / \mathrm{cmc}$ and $0.004 \%$ $\mathrm{S}-106 \mathrm{a} / \mathrm{cmc}$ reduced the surface tension of water more efficiently than pure surfactants of the same concentration (from 70.06 to $62.39 \mathrm{mN} / \mathrm{m}$ and 71.06 to $64.26 \mathrm{mN} / \mathrm{m}$ respectively). Stability of the alumina suspension was studied as well. The obtained results indicate that the addition of carboxymethylcellulose, surfactants, and the cmc-surfactant complexes leads to the increase of alumina suspension stability. The mechanism responsible for stabilization was electrosteric one. The largest stability was observed for the systems containing the mixture of cmc and surfactants. The reason for this is the formation of the adsorption layer composed of alternately arranged regions of polymer and surfactant complexes. Electrokinetic measurements of the studied systems allowed to describe the structure of the electrical double layer and to determine the point of zero charge of alumina $(\mathrm{pHpzc} \approx 7.5)$. The addition of the polymer as well as surfactants leads to the charge reversal of alumina.
\end{abstract}

Keywords Polysaccharides $\cdot$ Surfactants $\cdot$ Fluorosurfactants $\cdot$ Silicone $\cdot$ Adsorption $\cdot$ Carboxymethylcellulose

\section{Introduction}

Adsorption of polymers on different colloidal particles is a very important issue not only in industry but also in science [1]. After the addition of polymer to the studied system, its properties such as rheology and stability change $[2,3]$. There are several studies describing the process of synthetic and natural polymer adsorption on the solid surfaces [4-8]. It is a known fact that the systems containing oxide suspensions are widely used in different branches of industry. This paper focuses on the properties of alumina suspensions in the presence

Elżbieta Grządka

egrzadka@wp.pl

1 Department of Radiochemistry and Colloid Chemistry, Faculty of Chemistry, Maria Curie-Skłodowska University, Maria Curie-Skłodowska Sq. 3, 20-031 Lublin, Poland of anionic polysaccharide - carboxymethylcellulose $(\mathrm{cmc})$ and different cationic surfactants. Aluminum oxide $\left(\mathrm{Al}_{2} \mathrm{O}_{3}\right)$ is a naturally occurring mineral that can be found in several crystalline forms as well as in amorphous one. Owing to its high surface area and thermal stability as well as its hardness and mechanical strength, it has found numerous applications as a catalyst and adsorbent [9]. The paper by Goldberg et al. discusses thoroughly the surface properties of alumina including its different forms [10]. As follows from the abovementioned $\gamma-\mathrm{Al}_{2} \mathrm{O}_{3}$ is the most common crystalline form of aluminum oxide mostly due to its properties that are desirable in catalysis. Aqueous alumina suspensions draw interest of numerous scientists [11-14]. Different organic substances such as polymers can be used to change properties of oxide suspensions. Carboxymethylcellulose is an anionic polysaccharide containing negatively charged hydroxyl groups present in its chain [15]. Because of its properties, it is widely used in many branches of industry such as food, pharmaceuticals, medicine, or textiles [16]. Zhivkov and Hristov presented the data 
concerning the properties of the alumina suspension in the presence of carboxymethylcellulose [17]. They studied stability, adsorption, and electric properties of the carboxymethylcellulose $/ \gamma-\mathrm{Al}_{2} \mathrm{O}_{3}$ system using different techniques such as electric polarizability, mobility, and relaxation time, pointing out that the colloid stability is driven by the effective charge of cmc-alumina particles which is determined by different factors (surface charge, number of dissociated carboxylic groups of cmc chains, and the condensed counterions) [18]. However, there are only few investigations on the influence of surfactants on the polymer adsorption on the mineral oxide surfaces [19, 20]. In such case, the addition of surfactants to the polymer/ oxide suspensions can change their properties even to a greater extent. It is well known that the addition of surfactant molecules to the polymer solution leads to the formation of polymersurfactant complexes [21,22]. Such complexes found various interesting applications. Sharipova et al. described the use of the polymer-surfactant complexes in the stabilization and encapsulation of vitamin E [23]. In the previous paper, Grzadka investigated the effect of surfactants addition on the carrageenan adsorption on alumina establishing formation of polymersurfactant complexes between the oppositely charged species [24]. Shimabayshi and co-workers concluded that formation of the polymer-surfactant complexes and their influence on the stability of solid particle suspensions is complicated but very important to understand many biological issues [25].

According to the author's best knowledge, the influence of different kinds of surface active agents such as fluorinated and silicone surfactants on the adsorption, stability, and electrokinetic properties in the polymer/oxide systems was not discussed in the literature. Based on the available information about the properties of fluorinated surfactants [26], we believe that it is necessary to describe thoroughly the effect of such molecules on the abovementioned properties of colloidal systems. Fluorinated surfactants, also called fluorosurfactants, are amphiphilic molecules composed of a hydrophilic head bearing ionic or non-ionic charge and a hydrophobic chain composed of the alkyl chain in which the hydrogen atoms were replaced by fluorine $\left(-\mathrm{CF}_{\mathrm{x}}\right.$ instead of $\left.-\mathrm{CH}_{\mathrm{x}}\right)$. The presence of fluorine in the molecule results in higher chemical and thermal stability [27]. Due to the fact that hydrophobicity of fluorinated surfactants is much higher in comparison to the classical ones, they can reduce the surface tension of water to about $15-20 \mathrm{mN} \mathrm{m}^{-1}$ whereas hydrocarbon surfactants only to the value of $30-40 \mathrm{mN} \mathrm{m}^{-1}$ [28]. Despite the common opinion that fluorinated compounds are toxic and environmentally unfriendly [29], some patents claim that fluorinated surfactants can be used as dispersants for antihistamines, antibiotics, or antitussives aerosols as well as in the in vivo oxygen delivery systems [30]. Another interesting group of surface active agents is that of silicone surfactants. They are composed of a hydrophobic part soluble only in silicone and a hydrophilic part soluble in water. Due to the extensive use of silicone compounds in different formulations, it was important to discover silicone surfactants that can be used in a large variety of products. These surfactants can replace the conventional hydrocarbon surfactants especially in textile, coating, paint, and cosmetic industry [31].

The aim of this paper was to investigate the influence of cationic surfactants of different types (hydrocarbon, fluorinated, and silicone surfactants) on the adsorption of carboxymethylcellulose on the alumina surface as well as stability and electrokinetic properties of such systems using different methods (UV-Vis, DLS, potentiometric titration). Furthermore, interactions of all mentioned surfactants with carboxymethylcellulose were studied using the surface tension measurements.

\section{Materials and methods}

\section{Materials}

Gamma phase alumina $\left(\gamma-\mathrm{Al}_{2} \mathrm{O}_{3}\right)$ produced by Merck was used as the adsorbent. The specific surface area of this oxide was found to be $111 \mathrm{~m}^{2} \mathrm{~g}^{-1}$ (BET method), whereas the average pore size and the average pore volume of the adsorbent was $8.3 \mathrm{~nm}$ and $0.231 \mathrm{~cm}^{3} \mathrm{~g}^{-1}$, respectively. The adsorbent was washed with doubly distilled water until the conductivity of the supernatant was lower than $2 \mu \mathrm{Scm}^{-1}$. X-ray fluorescence spectroscopy confirmed that the alumina is impurity free. The analyzed sample contained Al $(97.930 \%)$, P (1.439\%), $\mathrm{Fe}(0.425 \%), \mathrm{Cl}(0.053 \%)$, Ti $(0.050 \%)$, and $\mathrm{Ni}$ $(0.029 \%)$. The particle size distribution of alumina was measured using Mastersizer 2000 and was in the range from $(\mathrm{d}(0.1), 10 \%$ of the particles are smaller than this diameter) $3.79 \mu \mathrm{m}$ to $(\mathrm{d}(0.9), 90 \%$ of the particles are smaller than this diameter) $32.79 \mu \mathrm{m}$, with the volume average size $(\mathrm{d}(0.5)$, the median diameter where $50 \%$ of the size distribution is above and $50 \%$ is below this value) of $13.10 \mu \mathrm{m}$. The morphology of the adsorbent was studied using scanning electron microscope (SEM). Figure 1 shows irregular shape of the alumina particles. Anionic polysaccharide carboxymethylcellulose sodium salt from Sigma-Aldrich was used as the adsorbate. Such properties of the polymer as viscosity average molecular weight and degree of substitution were described in the previous paper [32]. The surfactants used in this study were quaternary polydimethylsiloxane Silquat J2 (Siltech Corp.), tetradecyltrimethylammonium bromide MTAB (SigmaAldrich), and fluoroalkylammonium chloride Chemguard S106A (Chemguard). For the purpose of this study, they are called SJ2, MTAB, and S-106A, respectively. The background electrolyte $\mathrm{NaCl}$ as well as $\mathrm{NaOH}$ and $\mathrm{HCl}$ were purchased from Avantor Performance Materials Poland. 


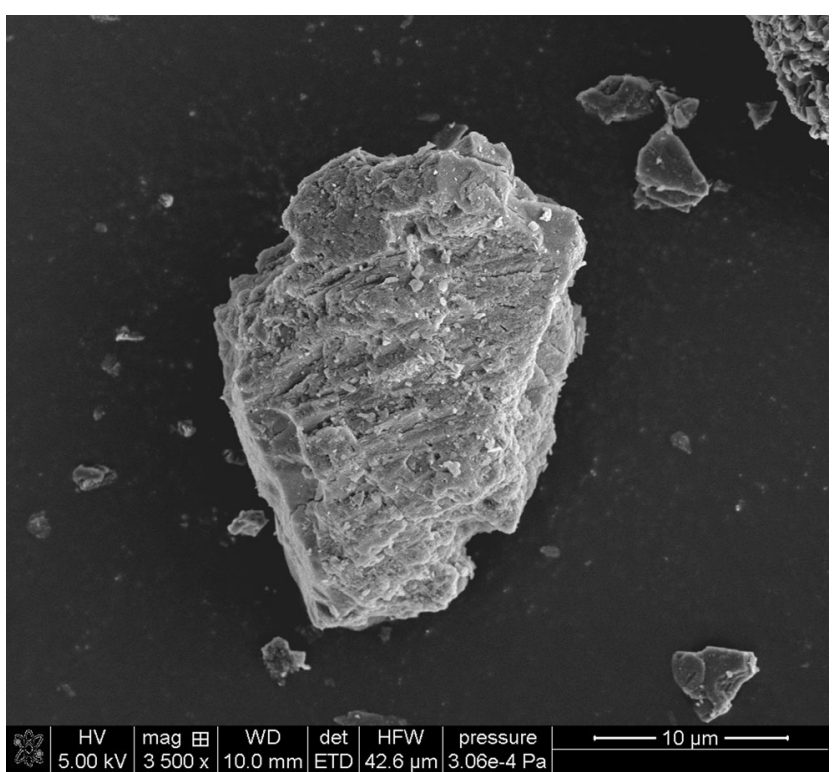

Fig. 1 SEM image showing the morphology of $\mathrm{Al}_{2} \mathrm{O}_{3}$

\section{Adsorption measurements}

In order to determine adsorption of cmc on the alumina surface, the modified Dubois colorimetric method described by Albalasmeh et al. was applied [33]. The calibration curve of the polysaccharide was made using different concentrations of the stock solution (20-600 ppm). The total volume of adsorption suspensions equals $10 \mathrm{~cm}^{3}$ and contains sodium chloride as a background electrolyte $\left(0.01 \mathrm{~mol} \mathrm{dm}^{-3}\right)$, defined volume of carboxymethylcellulose and $0.1 \mathrm{~g}$ of aluminum oxide. The $\mathrm{pH}$ of the obtained suspension was adjusted to 7 using $\mathrm{NaOH}$ or $\mathrm{HCl}$. Then the flasks with the suspensions were placed in the water bath while shaking $\left(25^{\circ} \mathrm{C}, 120 \mathrm{rpm}\right)$ over night (OLS 2000, Grant). The next day, the suspensions were centrifuged (15 min, $14.000 \mathrm{rpm}$ ) and the supernatants were used in the spectrophotometric measurements. The absorbance was

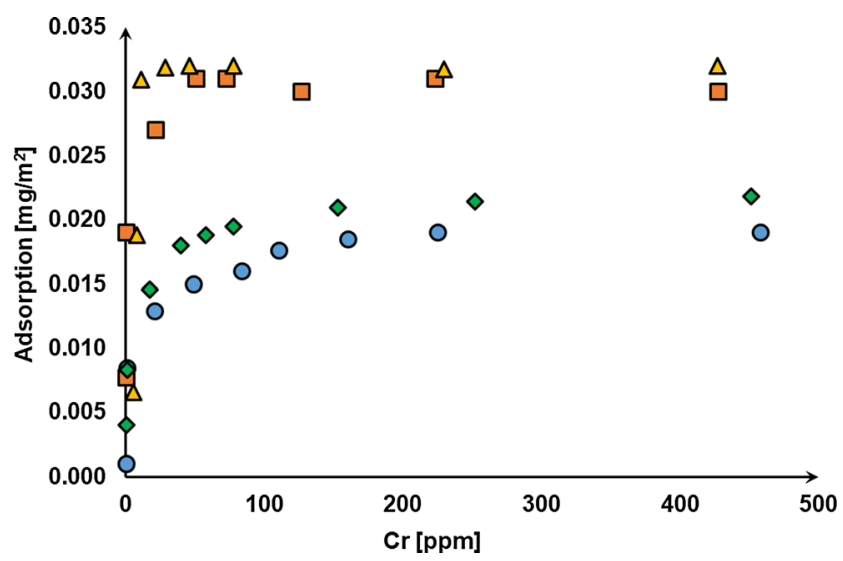

Fig. 2 Influence of different surfactants $(c=0.013 \%)$ on the adsorption of carboxymethylcellulose on the alumina surface: MTAB $+\mathrm{cmc}$ (triangle), $\mathrm{SJ} 2+\mathrm{cmc}$ (square), $\mathrm{S}-106 \mathrm{~A}+\mathrm{cmc}$ (rhomb), and pure cmc (circle); $\mathrm{pH}=$ 7, $0.01 \mathrm{~mol} \mathrm{dm}^{-3} \mathrm{NaCl}$ measured at $315 \mathrm{~nm}$ with a UV-Vis spectrophotometer (Cary 100 , Varian Instruments). In the case of the systems containing surfactants, the procedure was similar. For the solutions containing surfactants, different calibration curve was made. The concentration of the surface active agents used in this study was $0.013 \%(v / v)$. This concentration was chosen in order to avoid exceeding the critical micelle concentration values (CMC). All measurements were performed four times and the average values are reported.

\section{Stability measurements}

Determination of stability of the $\mathrm{cmc} / \mathrm{Al}_{2} \mathrm{O}_{3}$ and cmc/surfac$\operatorname{tant} / \mathrm{Al}_{2} \mathrm{O}_{3}$ systems was made using the UV-Vis spectrophotometer. The final volume of the suspension containing carboxymethylcellulose and sodium chloride was $10 \mathrm{~cm}^{3}$. The $\mathrm{Al}_{2} \mathrm{O}_{3}$ sample weight was $0.005 \mathrm{~g}$. The prepared suspensions were sonicated for $30 \mathrm{~s}$ and $\mathrm{pH}$ was adjusted to 7 . Then the samples were placed in the spectrophotometer chamber. The spectrum of the sample was collected in 300-800 $\mathrm{nm}$ range for the next $15 \mathrm{~h}$. The obtained results are shown in the form of the absorbance graphs in a function of time. All measurements were done as triplicates and the average values are reported.

\section{Zeta potential measurements}

A $0.1 \mathrm{~g}$ of aluminum oxide was added to $100 \mathrm{~cm}^{3}$ of background electrolyte solution $\left(\mathrm{NaCl}, 0.01 \mathrm{~mol} \mathrm{dm}^{-3}\right)$. After 3 min of sonication, in order to obtain the desired concentration $(1 \mathrm{ppm})$, the polymer stock solution was added. The polysaccharide was added after the sonication process to avoid polymer chains destruction. The electrophoretic mobility was measured using a zetameter (Zetasizer Nano ZS, Malvern Instruments) and the electrokinetic potential was calculated from the Smoluchowski equation [34]. The obtained data are shown in the form of graphs presenting the changes of the zeta potential in the $\mathrm{pH}$ function. In the case of the suspensions containing surfactants, the procedure was similar to that described above. The surface active agent was added together with the polymer after the ultrasonication so its final concentration was $0.013 \%$.

\section{Potentiometric titration}

Surface charge density of $\mathrm{Al}_{2} \mathrm{O}_{3}$ in the presence and absence of $\mathrm{cmc}$ and surfactants was determined using the potentiometric titration method. The $\mathrm{NaCl}$ concentration was $0.01 \mathrm{~mol} \mathrm{dm}^{-3}$. A thermostated, Teflon vessel with a shaker, an automatic burette (Dosimat 665, Methrom), and a pH meter were the parts of the measurement set. The process was controlled by a computer. The density of $\mathrm{Al}_{2} \mathrm{O}_{3}$ surface charge was determined using the "Miar_t" program written by 
Fig. 3 Schematic illustration showing the formation of polymer-surfactant multilayer on the alumina surface

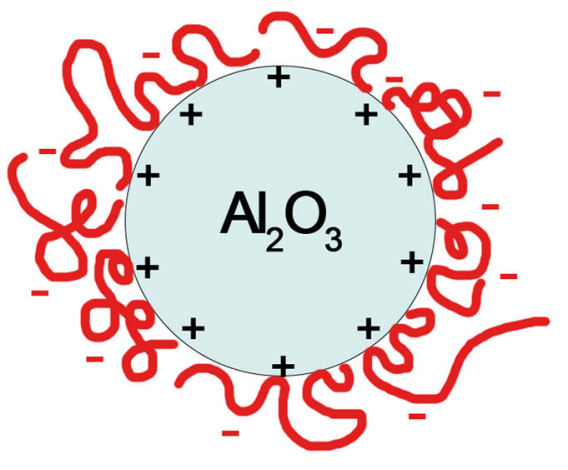

Janusz. The weight of the solid sample used in the measurements was $0.1 \mathrm{~g}$.

\section{Surface tension measurements}

In order to determine the interactions between carboxymethylcellulose and surfactants, surface tension measurements were conducted using the pendant drop method (Attension Theta optical tensiometer, KSV Instruments). The results are shown in the form of the changes of surface tension in the function of surfactant concentration. Aqueous solutions of surfactants with the increasing concentrations were prepared by dilution of the stock solution. To study the interactions between the polymer and surfactants, the samples were prepared in an analogous way but the carboxymethylcellulose stock solution was added in order to maintain the final polymer concentration of $400 \mathrm{ppm}$.

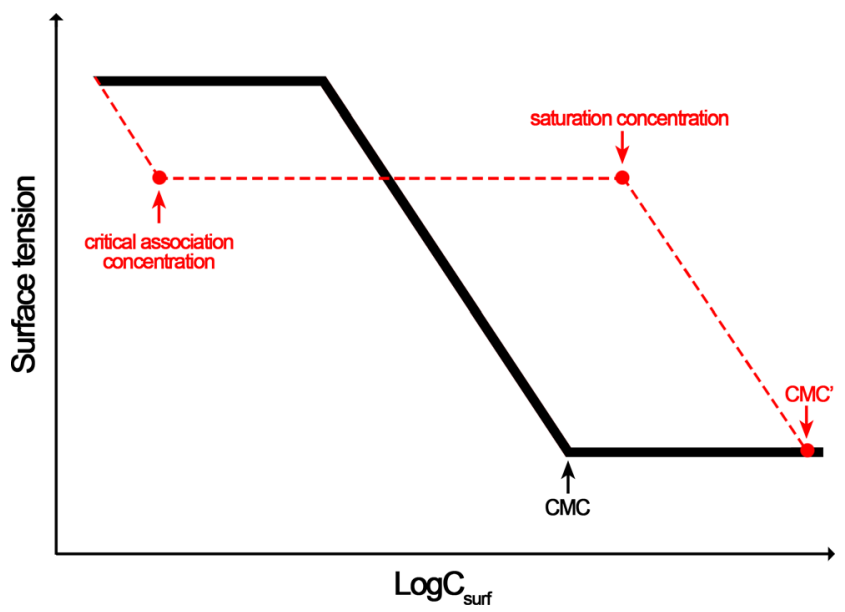

Fig. 4 Changes of the surface tension of surfactant solution (solid line) in the presence of polymer (dotted line) with visible transition pointscritical association concentration (T1), saturation concentration (T2), critical micelle concentration of pure surfactant (CMC), and critical micelle concentration of surfactant in the presence of polymer (CMC')

\section{Results and discussion}

The first step of this study was to investigate whether the adsorption of carboxymethylcellulose $(\mathrm{cmc})$ on the alumina surface is possible. Figure 2 shows the adsorption isotherm of $\mathrm{cmc}$ in the absence and presence of surfactants.

It can be seen that carboxymethylcellulose adsorbs on the alumina surface. In the case of polysaccharide adsorption, there are several mechanisms responsible for this process. The most important are hydrogen bonding, van der Waals interactions and acid-base interactions, etc. [35]. The aluminum(III) oxide adsorption groups depend on $\mathrm{pH}$ of the environment. According to Kosmulski's research [36, 37], the point of zero charge of $\gamma-\mathrm{Al}_{2} \mathrm{O}_{3}$ varies from 6.2 to about 9. In our case, the point of zero charge $\left(\mathrm{pH}_{\mathrm{pzc}}\right)$ equals 7.5. Below this $\mathrm{pH}$ value, the number of positively charged surface groups $\left(\mathrm{AlOH}_{2}^{+}\right)$is higher than that of the negative counterparts $\left(\mathrm{AlO}^{-}\right)$. Above this $\mathrm{pH}$ value, the surface is negatively charged. Due to the fact that the used polymer is of anionic character, its adsorption at $\mathrm{pH}=7$ proceeds mostly through the electrostatic interactions between the positively charged adsorbent and the negatively charged adsorbate. In such case, positive $\mathrm{AlOH}_{2}{ }^{+}$groups are covered by the cmc mono-layer causing their charge reversal. According to Rojas and Neuman, in the case of the oppositely charged adsorbate and adsorbent, electrostatic (ionic) interactions occurs [38]. However, the additional role in the adsorption mechanism can be played by hydrogen bonding between the functional groups of cmc and numerous hydroxyl groups present on the $\mathrm{Al}_{2} \mathrm{O}_{3}$ surface $[39,40]$. Thus, such intermolecular interactions should also be taken into account as possible adsorption mechanisms. Moreover, it can be observed that the adsorption of carboxymethylcellulose increases after the addition of surfactants. This is due to the formation of the adsorption multilayer composed of polymer-surfactant complexes. In this case, the affinity of such complex for the alumina surface is higher than for the pure polymer. Figure 3 shows the schematic formation of polymer-surfactant multilayer. 
Fig. 5 Tensiometric studies of the interactions between

carboxymethylcellulose $(\mathrm{cmc}$,

$400 \mathrm{ppm}$ ) and different

surfactants: a hydrocarbon

MTAB (rhomb), MTAB/cmc

(circle); b silicone SJ2 (rhomb),

$\mathrm{SJ} 2 / \mathrm{cmc}$ (circle); c fluorinated

S-106A (rhomb), S-106A/cmc

(circle)
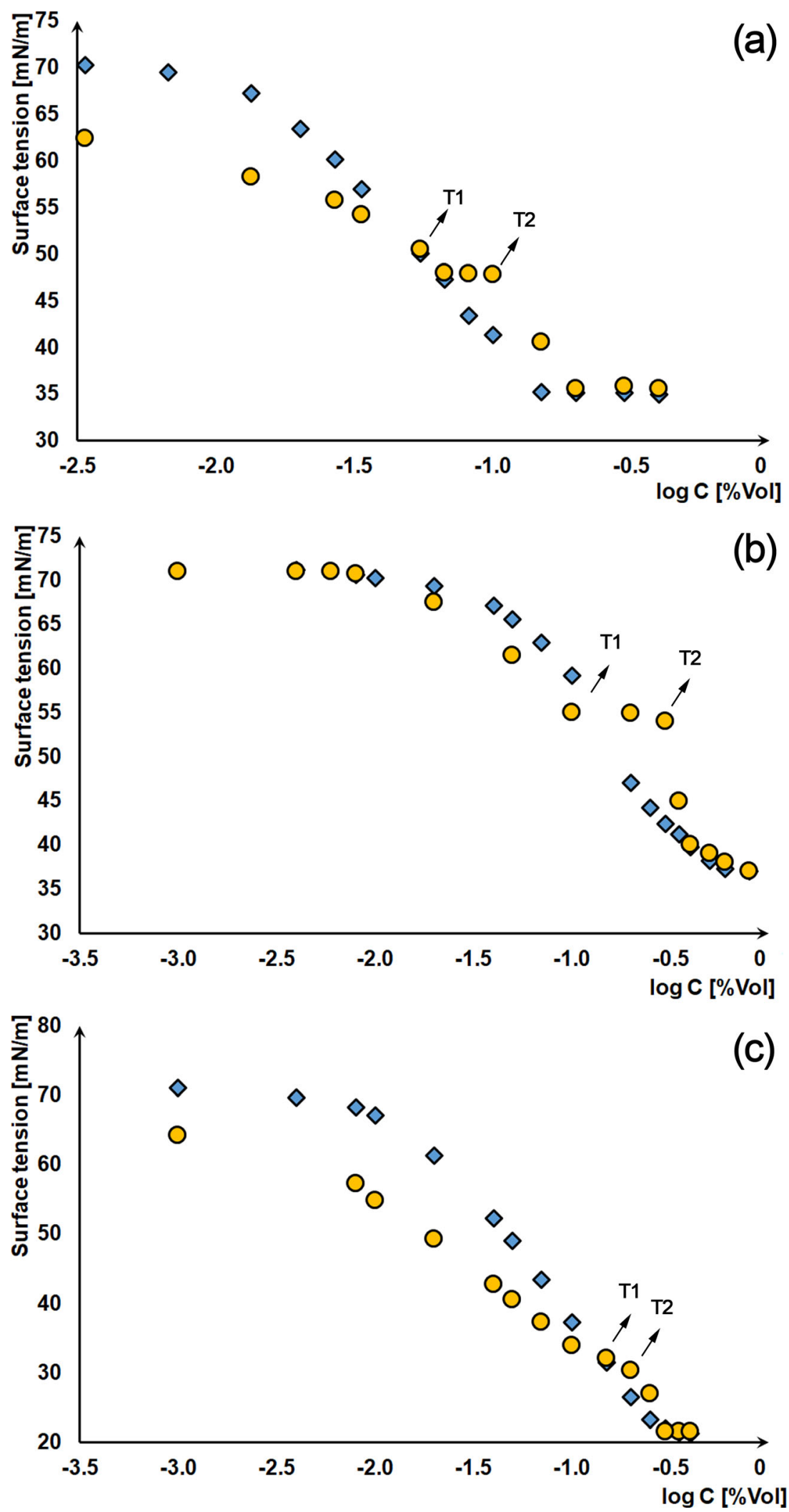

To confirm formation of such multilayer, the tensiometric studies of the polymer-surfactant interactions were carried out. There are a few transition points that should be recalled in the case of polymer-surfactants interactions (Fig. 4) [41]. The first one is the point called the critical association concentration (CAC, T1). It is the lowest 
Fig. 6 Influence of surfactants $(0.013 \%)$ and

carboxymethylcellulose $(\mathrm{cmc}$, $400 \mathrm{ppm}$ ) as well as its mixtures on stability of the alumina suspension: a no additives (cross), MTAB (circle), cmc (square), cmc + MTAB (rhomb); b no additives (cross), SJ2 (circle), cmc (square), cmc + SJ2 (rhomb); c no additives (cross), S-106A (circle), cmc (square), $\mathrm{cmc}+\mathrm{S}-106 \mathrm{~A}$ (rhomb); $\mathrm{pH}=7, C_{\mathrm{NaCl}}=$ $0.01 \mathrm{~mol} \mathrm{dm}^{-3}$
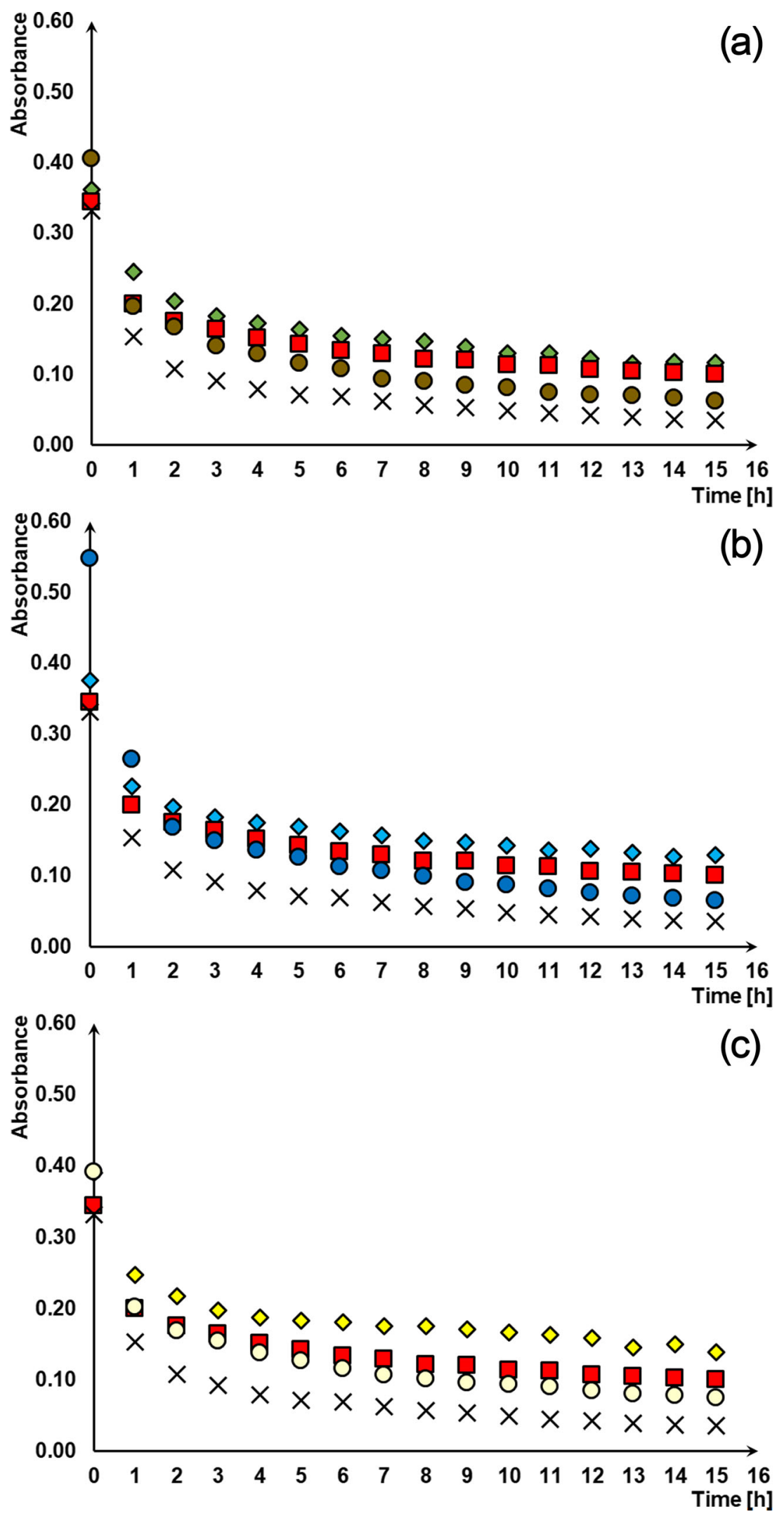

concentration of surfactant at which the interactions between the polymer chains and the surfactant molecules start. A further increase of surfactant concentration leads to the situation when the polymer chains are saturated with 


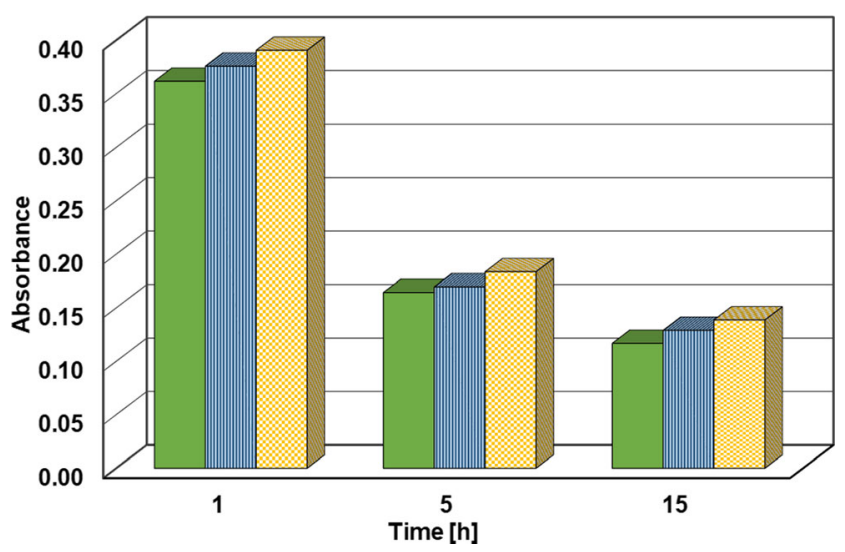

Fig. 7 Comparison of stability of alumina suspension in the presence of cmc-surfactant mixture (400 ppm + 0.013\%): MTAB + cmc (plain), SJ2 + cmc (stripes), $\mathrm{S}-106 \mathrm{~A}+\mathrm{cmc}$ (squares); $\mathrm{pH}=7, C_{\mathrm{NaCl}}=0.01 \mathrm{~mol} \mathrm{dm}^{-3}$

surfactant molecules. This point is called the saturation concentration (T2).

In all studied systems, formation of the polymer-surfactant complexes was observed. Figure 5 shows the changes in the surface tension of surfactants in the absence and presence of cmc. It can be noticed that the critical association concentration (T1) is visible in every case. Hence, it is possible to determine the point where the interactions between the polymer and the surfactant start. Critical association concentration of surfactants in the presence of carboxymethylcellulose equals $0.055 \%$ for $\mathrm{MTAB}, 0.1 \%$ for $\mathrm{SJ} 2$, and $0.15 \%$ for S-106A. Furthermore, the saturation concentration points (T2) are visible as well. In the case of MTAB and S-106A surfactants, an immediate decrease in the surface tension values after the addition of carboxymethylcellulose shows that the polymer-surfactant complex possesses higher surface activity than the pure surfactant solution [42].

For the silicone surfactant (SJ2), an immediate decrease of surface tension after the addition of $\mathrm{cmc}$ is not observed. This means that the formed complexes do not possess great ability to reduce surface tension of the solution. According to Taylor et al. the polymer-surfactant complexes of the specific concentration are completely soluble in water [43]. However, with the increasing surfactant concentration, precipitation of such complexes is observed. In the case of the presented results, it was confirmed that the $\mathrm{cmc} / \mathrm{MTAB}, \mathrm{cmc} / \mathrm{SJ} 2$, and $\mathrm{cmc} / \mathrm{S}$ 106A complexes were completely soluble in the smaller surfactant concentration range. Nonetheless, for the highest concentrations, the haze cloud-like structures were observed indicating that above certain concentration, the complexes started to be insoluble. The adsorption measurements of $\mathrm{cmc}$ in the presence of surfactants showed the highest adsorption for the systems containing $\mathrm{cmc} / \mathrm{MTAB}$ and $\mathrm{cmc} / \mathrm{SJ} 2$. Definitely lower adsorption was observed for the $\mathrm{cmc} / \mathrm{S}-106 \mathrm{~A}$ system. This indicates that in the case of hydrocarbon and silicone surfactants, the complexes with carboxymethylcellulose are formed very effectively. Hence the formed adsorption layer is thicker and the complexes possess higher affinity for the alumina surface than carboxymethylcellulose itself. Despite the fact that the cmc/S-106A complex has a higher surface activity than $\mathrm{cmc} / \mathrm{SJ} 2$, it seems that the fluorinated surfactant prefers to form associates with $\mathrm{cmc}$ that remain in the bulk solution rather than adsorb on the alumina surface.

To study the influence of surfactants on stability of the cmc/ $\mathrm{Al}_{2} \mathrm{O}_{3}$, the UV-Vis method was used. As one can see, the alumina suspensions are not stable. After a short period of time, the suspended particles tend to aggregate and sedimentation takes place. To counteract this undesirable problem, the addition of both carboxymethylcellulose and surfactants is proposed. It is known that the addition of polymer or surface active agent to the oxide suspension leads to its stabilization or destabilization. As far as stabilization is concerned, this process depends on the nature of the polymer or surfactant as well as its adsorptive properties. Different scenarios can be
Fig. 8 The zeta potential of alumina (cross) in the presence of cmc (circle, $1 \mathrm{ppm}$ ) and surfactants $(0.013 \%)$ : SJ2 (rhomb), S-106A (triangle), MTAB (square); $C_{\mathrm{NaCl}}=$ $0.01 \mathrm{~mol} \mathrm{dm}^{-3}$

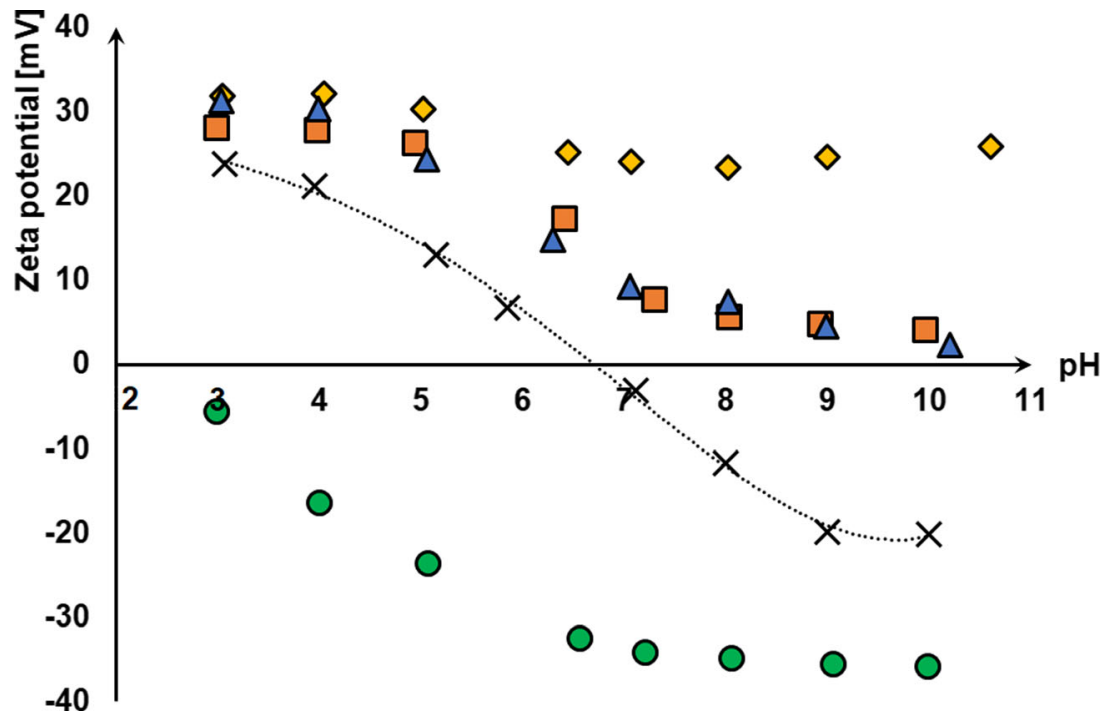


Fig. 9 Influence of cmc (circle, $400 \mathrm{ppm}$ ) and surfactants $(0.013 \%)$ : MTAB (square), SJ2 (rhombus), S-106A (triangle) on the surface charge density of $\mathrm{Al}_{2} \mathrm{O}_{3}$ in the presence of $0.01 \mathrm{~mol} \mathrm{dm}^{-3} \mathrm{NaCl}$

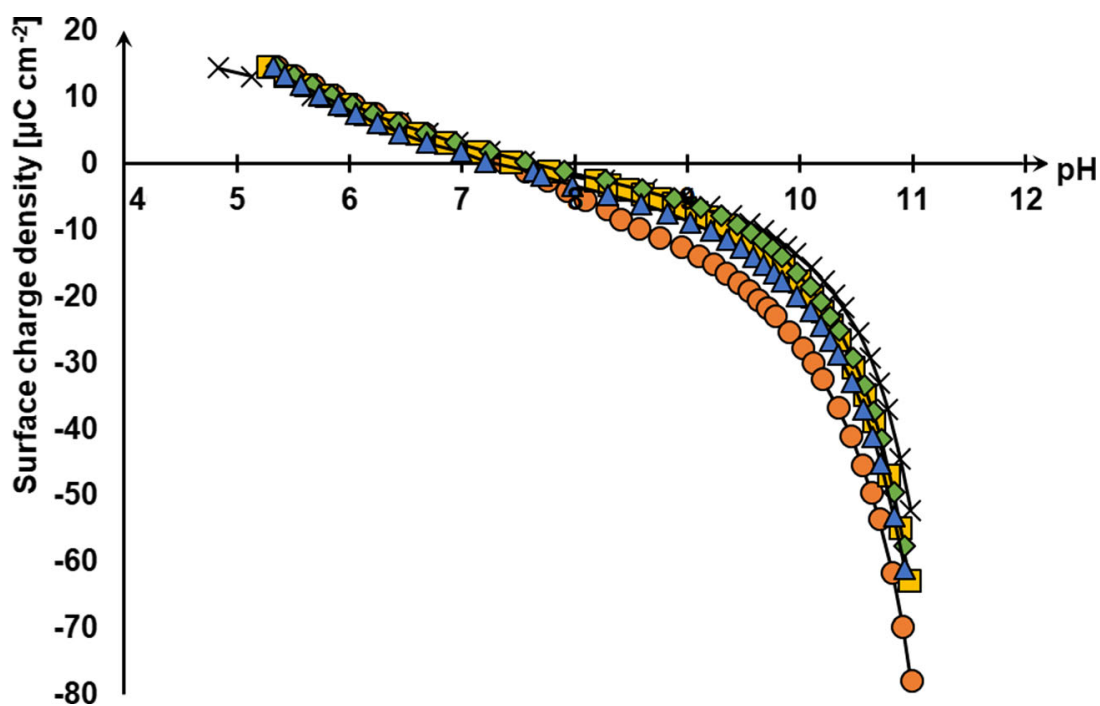

distinguished: for example, steric, electrosteric, and depletion stabilization. These phenomena were profoundly discussed in the literature [44-47]. Figure 6 shows the changes in the alumina suspension stability in the presence and absence of polymer, surfactant, and polymer-surfactant mixture. It can be seen that the addition of carboxymethylcellulose, surfactants, and cmc-surfactant complexes leads to the increase of stability of the alumina suspension. Due to the fact that polysaccharide as well as the surfactants are characterized by ionic character and can adsorb on $\mathrm{Al}_{2} \mathrm{O}_{3}$, the mechanism responsible for stabilization was electrosteric one. In the case of $\mathrm{cmc} / \mathrm{MTAB} / \mathrm{Al}_{2} \mathrm{O}_{3}$, $\mathrm{cmc} / \mathrm{SJ} 2 / \mathrm{Al}_{2} \mathrm{O}_{3}$, and $\mathrm{cmc} / \mathrm{S}-106 \mathrm{~A} / \mathrm{Al}_{2} \mathrm{O}_{3}$, a similar situation was observed. The lowest increase of stability was observed in the presence of pure surfactant. This is most likely due to the fact that the surfactant molecules present in the solution adsorb on the alumina particles covering its surface. However, such layer is not as dense as in the case of polymer adsorption. That is why higher stability was observed for the systems containing the high molecular weight substance. In this case, the adsorption layer formed on the solid particles is thicker. The highest stability was observed for the systems containing the mixture of cmc and surfactants. The reason for this is formation of the adsorption layer composed of alternately arranged regions of polymer and surfactant complexes.

The comparison of alumina suspension stability in the presence of the polymer-surfactant mixtures is shown in Fig. 7. In this case, the highest stability was observed for the mixture of fluorinated surfactant S-106A and cmc. Even though the adsorption of cmc in the presence of S-106A is lower than in the case of other surfactants, the formed adsorption layer can be thicker and more expanded towards the bulk of the solution. Hence, in such case, the highest stability of the alumina suspension is observed. Nonetheless, all of the studied mixtures increased the stability of the alumina suspensions and the differences between them were minimal.
To study electrokinetic properties of the systems, electrophoretic mobility measurements were performed. The obtained data were recalculated to the zeta potential using the Smoluchowski equation. Figure 8 shows the changes in the zeta potential in the $\mathrm{pH}$ function for the studied systems.

Based on the collected data, it was possible to determine the $\mathrm{pH}_{\text {iep }}$ of alumina. For this adsorbent, the isoelectric point was estimated at $\mathrm{pH}$ about 7.5. That is why below this value, the alumina surface is positively charged. The addition of $1 \mathrm{ppm}$ of cmc leads to complete charge reversal of the alumina. Due to the adsorption of carboxymethylcellulose and its high surface charge density, the solid particles are tightly covered with the polymer chains. This leads to the situation in which the slipping plane is moved towards the bulk solution resulting in the decrease of zeta potential values. Furthermore, the negatively charged polymer groups coming from its dissociation are present in the diffused part of the electrical double layer (edl). It can be observed that in the case of the $\mathrm{cmc} /$ $\mathrm{Al}_{2} \mathrm{O}_{3}$ system, the zeta potential stabilizes at the $\mathrm{pH}$ value above 8 . This is most likely due to the fact that the total negative charge is high. In the situation when the zeta potential is about $-35 \mathrm{mV}$, it can be stated that the studied system is stable [48]. The surfactants addition to the $\mathrm{cmc} / \mathrm{Al}_{2} \mathrm{O}_{3}$ system leads to the positive increase of the zeta potential. It should be emphasized that both polymer and surfactants adsorb on the alumina surface. The observed increase in the zeta potential (more positive values) is the result of positively charged surfactant groups present in the diffused part of the electrical double layer. In all three cases, the complete charge reversal caused by the surfactant molecules is observed. The most positive zeta potential values are observed for the $\mathrm{cmc} / \mathrm{SJ} 2 /$ $\mathrm{Al}_{2} \mathrm{O}_{3}$. This is most likely due to the fact that this silicone surfactant has the highest positive surface charge which affects the zeta potential of suspension. 
The potentiometric titration method allows calculating and observing the changes of surface charge density of the solid in the presence and absence of the polymer and surfactants.

Figure 9 presents the influence of cmc and surfactants on the surface charge density of $\mathrm{Al}_{2} \mathrm{O}_{3}$. As it was mentioned before, the surface charge of alumina depends largely on $\mathrm{pH}$. The presence of negatively charged cmc leads to the decrease of $\mathrm{Al}_{2} \mathrm{O}_{3}$ surface charge. The reason for this is the presence of anionic groups in the compact surface layer of alumina. The addition of surfactants to the systems causes a slight increase of the surface charge. This is most likely caused by the presence of positively charged surfactant groups, not bound with the alumina surface, present in the surface layer of $\mathrm{Al}_{2} \mathrm{O}_{3}$.

\section{Conclusions}

The studies prove that both conventional and unconventional surfactants can interact with carboxymethylcellulose, which leads to formation of the polymer-surfactant complexes. Fluorinated (S-106A), silicone (SJ2), and hydrocarbon (MTAB) surfactants have the impact on the adsorption of carboxymethylcellulose on the alumina surface. Furthermore, the highest adsorption of carboxymethylcellulose was observed in the presence of surfactants: hydrocarbon MTAB (0.03$0.032 \mathrm{mg} \mathrm{m}^{-2}$ ) and silicone SJ2 (0.027-0.03 $\left.\mathrm{mg} \mathrm{m}^{-2}\right)$. The reason for this is the effective formation of complex adsorption multilayer composed of polymer chains and surfactant molecules. The interactions between the surfactants and cmc were confirmed by the tensiometric measurements. The cmc/MTAB and $\mathrm{cmc} / \mathrm{S}-106 \mathrm{a}$ complexes were characterized by larger surface activity than pure surfactants. The decrease of the surface tension of water of MTAB and S-106a solutions after the addition of carboxymethylcellulose by about $8 \mathrm{mN} / \mathrm{m}$ was observed (for the lowest concentrations of surfactants). In the case of cmc/SJ2, the surface activity of formed complex and pure surfactant was similar. Nonetheless, in the case of cmcsurfactants complexes, the transition points such as critical association concentration (T1) and saturation concentration (T2) were observed $(\mathrm{MTAB} \mathrm{T} 1=0.055 \%, \mathrm{~T} 2=0.1 \%$; SJ2 $\mathrm{T} 1=$ $0.1 \%, \mathrm{~T} 2=0.3 \%$, and S-106a $\mathrm{T} 1=0.15 \%, \mathrm{~T} 2=0.2 \%)$. The studies of alumina suspensions stability in the presence and absence of cmc, surfactants, and their mixtures indicate that the highest stability was observed for the cmc/surfactant/ $\mathrm{Al}_{2} \mathrm{O}_{3}$ system. This is due to the fact that the formed adsorption multilayer leads to reduced interactions between the solid particles resulting in higher system stability. The mechanism responsible for that is the electrosteric stabilization. Electrokinetic measurements of the studied systems allowed the description of the structure of edl. In the presence of cmc, the alumina surface charge is reversed because of the adsorption of negatively charged polymer chains on its surface as well as its presence in the diffused part of edl. The addition of surfactants to the $\mathrm{cmc} / \mathrm{Al}_{2} \mathrm{O}_{3}$ system leads to the charge reversal as well. The highest (positive) zeta potential is observed for the $\mathrm{SJ} 2 / \mathrm{cmc} / \mathrm{Al}_{2} \mathrm{O}_{3}$ system (the range 31.8 $25.8 \mathrm{mV}$ ). This is most likely due to its highest positive charge input. The presented results show that both silicone and fluorinated surfactants can be used as an alternative in different colloidal formulations and areas for example in the functionalization of hybrid materials.

Acknowledgments Authors would like to thank Brandon Lines from Siltech Corporation (Toronto, Canada) as well as Audrey Bielat from Chemguard (Marinette, USA) for the delivery of surfactant samples used in this study.

Funding information This study received financial support from the Polish Ministry of Science and Higher Education for the Faculty of Chemistry of Maria Curie Skłodowska University in Lublin under statutory activity subsidies.

\section{Compliance with ethical standards}

Conflict of interest The authors declare that they have no conflict of interest.

Open Access This article is distributed under the terms of the Creative Commons Attribution 4.0 International License (http:// creativecommons.org/licenses/by/4.0/), which permits unrestricted use, distribution, and reproduction in any medium, provided you give appropriate credit to the original author(s) and the source, provide a link to the Creative Commons license, and indicate if changes were made.

\section{References}

1. Llamas S, Guzmán E, Ortega F, Baghdadli N, Cazeneuve C, Rubio RG, Luengo GS (2015) Adsorption of polyelectrolytes and polyelectrolytes-surfactant mixtures at surfaces: a physicochemical approach to a cosmetic challenge. Adv Colloid Interf Sci 222:461-487. https://doi.org/10.1016/j.cis.2014.05.007

2. Borkovec M, Papastavrou G (2008) Interactions between solid surfaces with adsorbed polyelectrolytes of opposite charge. Curr Opin Colloid Interface Sci 13:429-437. https://doi.org/10.1016/j.cocis. 2008.02.006

3. Szilagyi I, Trefalt G, Tiraferri A, Maroni P, Borkovec M (2014) Polyelectrolyte adsorption, interparticle forces, and colloidal aggregation. Soft Matter 10:2479-2502. https://doi.org/10.1039/ C3SM52132J

4. Mészáros R, Varga I, Gilányi T (2004) Adsorption of poly(ethyleneimine) on silica surfaces. Langmuir 20:5026-5029. https://doi.org/10.1021/la0496111

5. Chibowski S, Grządka E, Patkowski J (2008) Comparison of the influence of a kind of electrolyte and its ionic strength on the adsorption and electrokinetic properties of the interface: polyacrylic acid/MnO2/electrolyte solution. Colloids Surf A Physicochem Eng Asp 326:191-203. https://doi.org/10.1016/j.colsurfa.2008.05.038

6. Ostolska I, Wiśniewska M (2014) Comparison of the influence of polyaspartic acid and polylysine functional groups on the adsorption at the $\mathrm{Cr} 2 \mathrm{O} 3$-aqueous polymer solution interface. Appl Surf Sci 311:734-739. https://doi.org/10.1016/j.apsusc.2014.05.149 
7. Matusiak J, Grządka E, Bastrzyk A (2018) Stability, adsorption and electrokinetic properties of the chitosan/silica system. Colloids Surf A Physicochem Eng Asp 554:245-252. https://doi.org/10.1016/j. colsurfa.2018.06.056

8. Gozdecka A, Wiacek AE (2018) Effect of UV radiation and chitosan coating on the adsorption-photocatalytic activity of $\mathrm{TiO} 2$ particles. Mater Sci Eng C 93:582-594. https://doi.org/10.1016/j.msec. 2018.08.022

9. Kasprzyk-Hordern B (2004) Chemistry of alumina, reactions in aqueous solution and its application in water treatment. Adv Colloid Interf Sci 110:19-48. https://doi.org/10.1016/j.cis.2004.02. 002

10. Goldberg S, Davis JA, Hem JD (1996) The surface chemistry of aluminum oxides and hydroxides. In: Sposito G (ed) The environmental chemistry of aluminum2nd edn. CRC Press, Boca Raton, pp 271-318

11. Akhlaghi O, Akbulut O, Menceloglu YZ (2015) Extensional rheology and stability behavior of alumina suspensions in the presence of AMPS-modified polycarboxylate ether-based copolymers. Colloid Polym Sci 293:2867-2876. https://doi.org/10.1007/s00396-0153683-8

12. Vasconcelos de CL, Dantas TNC, Pereira MR, Fonseca JLC (2004) Rheology of concentrated alumina-polyelectrolyte systems. Colloid Polym Sci 282:596-601. https://doi.org/10.1007/s00396-003-09840

13. Hidber PC, Graule TJ, Gauckler LJ (1997) Influence of the dispersant structure on properties of electrostatically stabilized aqueous alumina suspensions. J Eur Ceram Soc 17:239-249. https://doi.org/ 10.1016/S0955-2219(96)00151-3

14. Ringebach E, Chauveteau G, Pefferkom E (1995) Effect of soluble aluminum ions on polyelectrolyte-alumina interaction. Kinetics of polymer adsorption and colloid stabilization. Colloids Surf A Physicochem Eng Asp 99(1-2):161-173. https://doi.org/10.1016/ 0927-7757(95)03152-4

15. Wang J, Somasundaran P (2005) Adsorption and conformation of carboxymethyl cellulose at solid-liquid interfaces using spectroscopic, AFM and allied techniques. J Colloid Interface Sci 291: 75-83. https://doi.org/10.1016/j.jcis.2005.04.095

16. Zhao X, Clifford A, Poon R, Mathews R, Zhitomirsky I (2018) Carboxymethyl cellulose and composite films prepared by electrophoretic deposition and liquid-liquid particle extraction. Colloid Polym Sci 296:927-934. https://doi.org/10.1007/s00396-0184314-y

17. Zhivkov AM, Hristov RP (2015) Adsorption of caboxymethyl cellulose on alumina particles. J Colloid Interface Sci 447:159-166. https://doi.org/10.1016/j.jcis.2014.11.051

18. Zhivkov AM, Hristov RP (2017) Stability of aqueous suspensions of alumina particles with adsorbed (carboxymethyl)cellulose. Colloids Surf A Physicochem Eng Asp 529:523-530. https://doi. org/10.1016/j.colsurfa.2017.06.037

19. Grzadka E (2013) Influence of surfactants on the adsorption and elektrokinetic properties of the system: guar gum/manganese dioxide. Cellulose 20:1313-1328. https://doi.org/10.1007/s10570-0139902-x

20. Grzadka E, Matusiak J (2017) The effect of ionic and non-ionic surfactants and $\mathrm{pH}$ on the stability, adsorption and electrokinetic properties of the alginic acid/alumina system. Carbohydr Polym 175:192-198. https://doi.org/10.1016/j.carbpol.2017.07.066

21. Nagarajan R, Kalpakci B (1985) Viscometric investigation of complexes between polyethyleneoxide and surfactant micelles. In: Dubin P (ed) Microdomains in polymer solutions. Polymer science and technology, vol 30. Springer, Boston, pp 369-381

22. La Mesa C (2005) Polymer-surfactant and protein-surfactant interactions. J Colloid Interface Sci 286:148-157. https://doi.org/10. 1016/j.jcis.2004.12.038
23. Sharipova AA, Aidarovaa SB, Grigoriev B, Mutalieva B, Madibekova G, Tleuova A, Miller R (2016) Polymer-surfactant complexes for microencapsulation of vitamin $\mathrm{E}$ and its release. Colloids Surf B 137:152-157. https://doi.org/10.1016/j.colsurfb. 2015.03.063

24. Grządka E (2015) Interactions between kappa-carrageenan and some surfactants in the bulk solution and at the surface of alumina. Carbohydr Polym 123:1-7. https://doi.org/10.1016/j.carbpol.2015. 01.024

25. Shimabayashi S, Uno T, Nakagaki M (1997) Formation of a surface complex between polymer and surfactant and its effect on the dispersion of solid particles. Colloids Surf A Physicochem Eng Asp 123-124:283-295. https://doi.org/10.1016/S0927-7757(96) 03820-4

26. Szymczyk K (2014) Comparative study of the physicochemical properties of aqueous solutions of the hydrocarbon and fluorocarbon surfactants and their ternary mixtures. Chem Phys 433:42-47. https://doi.org/10.1016/j.chemphys.2014.02.001

27. Kovalchuk NM, Trybala A, Starov V, Matar O, Ivanova N (2014) Fluoro- vs hydrocarbon surfactants: why do they differ in wetting performance? Adv Colloid Interf Sci 210:65-71. https://doi.org/10. 1016/j.cis.2014.04.003

28. Pabon M, Corpart JM (2002) Fluorinated surfactants: synthesis, properties, effluent treatment. J Fluor Chem 114:149-156. https:// doi.org/10.1016/S0022-1139(02)00038-6

29. Stahl T, Mattern D, Brunn H (2011) Toxicology of perfluorinated compounds. Environ Sci Eur 23(38). https://doi.org/10.1186/21904715-23-38

30. Krafft MP (2001) Fluorocarbons and fluorinated amphiphiles in drug delivery and biomedical research. Adv Drug Deliv Rev 47: 209-228. https://doi.org/10.1016/S0169-409X(01)00107-7

31. O'Lenick AJ (2000) Silicone emulsions and surfactants. J Surfactant Deterg 3:387-393. https://doi.org/10.1007/s11743-0000143-y

32. Grządka E, Chibowski S (2012) Adsorption and elektrokinetic properties of the system: carboxymethylcellulose/manganese oxide/surfactant. Cellulose 19:23-36. https://doi.org/10.1007/ s10570-011-9611-2

33. Albalasmeh AA, Berhe AA, Ghezzehei TA (2013) A new method for rapid determination of carbohydrate and total carbon concentrations using UV spectrophotometry. Carbohydr Polym 97(2):253261. https://doi.org/10.1016/j.carbpol.2013.04.072

34. Zembala M (2004) Electrokinetics of heterogeneous interfaces. Adv Colloid Interf Sci 112:59-92. https://doi.org/10.1016/j.cis. 2004.08.001

35. Laskowski JS, Liu Q, O’Connor CT (2007) Current understanding of the mechanism of polysaccharide adsorption at the mineral/ aqueous solution interface. Int J Miner Process 84(1-4):59-68. https://doi.org/10.1016/j.minpro.2007.03.006

36. Kosmulski M (2009) $\mathrm{pH}$-dependent surface charging and points of zero charge. IV. Update and new approach. J Colloid Interface Sci 337:439-448. https://doi.org/10.1016/j.jcis.2009.04.072

37. Kosmulski M (2001) Chemical properties of material surfaces. Marcel Dekker, New York

38. Rojas OJ, Neuman RD (1999) Adsorption of polysaccharide wetend additives in papermaking systems. Colloids Surf A Physicochem Eng Asp 155:419-432. https://doi.org/10.1016/ S0927-7757(99)00040-0

39. Hoogendam CW, de Keizer A, Cohen Stuart MA, Bijsterbosch BH (1998) Adsorption mechanisms of carboxymethyl cellulose on mineral surfaces. Langmuir 14:3825-3839. https://doi.org/10.1021/ la9800046

40. Bicak O, Ekmekci Z, Bradshow DJ, Harris PJ (2007) Adsorption of guar gum and CMC on pyrite. Miner Eng 20(10):996-1002. https:// doi.org/10.1016/j.mineng.2007.03.002 
41. Tajik B, Sohrabi B, Amani R, Hashemianzadeh SM (2013) The study of polymer-surfactant interaction in cationic surfactant mixtures. Colloids Surf A Physicochem Eng Asp 436:890-897. https:// doi.org/10.1016/j.colsurfa.2013.07.026

42. Goddard ED (1986) Polymer-surfactant interaction part II. Polymer and surfactant of opposite charge. Colloids Surf A Physicochem Eng Asp 19:301-329. https://doi.org/10.1016/0166-6622(86) 80341-9

43. Taylor DJF, Thomas RK, Penfold J (2007) Polymer-surfactant interactions at the air/water interface. Adv Colloid Interf Sci 132:69110. https://doi.org/10.1016/j.cis.2007.01.002

44. Sato T, Ruch R (1980) Stabilization of colloidal dispersion by polymer adsorption. Marcel Dekker Inc, New York

45. Sung AM, Piirma I (1994) Electrosteric stabilization of polymer colloids. Langmuir 10:1393-1398. https://doi.org/10.1021/ la00017a014
46. Evnas R, Napper DH (1973) Steric stabilization I. KolloidZeitschrift und Zeitschrift für Polymere 251:409-414. https://doi. org/10.1007/BF01498687

47. Semenov AN, Shvets AA (2015) Theory of colloid depletion stabilization by unattached and adsorbed polymers. Soft Matter 11: 8863-8878. https://doi.org/10.1039/C5SM01365H

48. Ostolska I, Wiśniewska M (2014) Application of the zeta potential measurements to explanation of colloidal $\mathrm{Cr} 2 \mathrm{O} 3$ stability mechanism in the presence of the ionic polyaminoacids. Colloid Polym Sci 292:2453-2464. https://doi.org/10.1007/s00396-014-3276-y

Publisher's note Springer Nature remains neutral with regard to jurisdictional claims in published maps and institutional affiliations. 